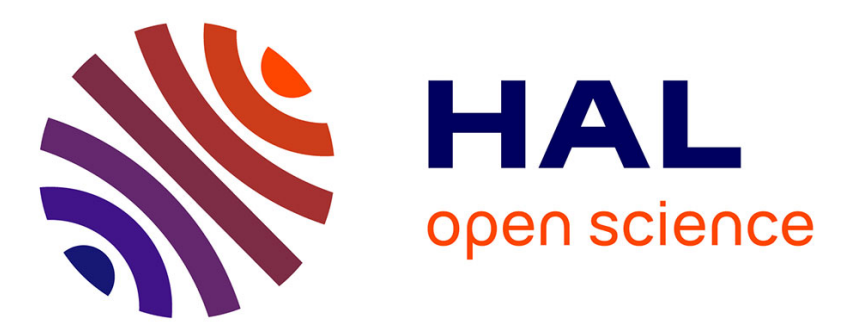

\title{
The average $\cos \delta$ approach to the theoretical calculation of thermal energy ion-dipolar molecule reaction rate constant
}

\author{
A.M. Kosmas
}

\section{> To cite this version:}

A.M. Kosmas. The average $\cos \delta$ approach to the theoretical calculation of thermal energy iondipolar molecule reaction rate constant. Journal de Physique Lettres, 1984, 45 (22), pp.1083-1089. 10.1051/jphyslet:0198400450220108300 . jpa-00232453

\section{HAL Id: jpa-00232453 https://hal.science/jpa-00232453}

Submitted on 1 Jan 1984

HAL is a multi-disciplinary open access archive for the deposit and dissemination of scientific research documents, whether they are published or not. The documents may come from teaching and research institutions in France or abroad, or from public or private research centers.
L'archive ouverte pluridisciplinaire HAL, est destinée au dépôt et à la diffusion de documents scientifiques de niveau recherche, publiés ou non, émanant des établissements d'enseignement et de recherche français ou étrangers, des laboratoires publics ou privés. 
Classification

Physics Abstracts

34.50L

\title{
The average $\cos \vartheta$ approach to the theoretical calculation of thermal energy ion-dipolar molecule reaction rate constant
}

\author{
A. M. Kosmas \\ Department of Chemistry, University of Ioannina, Greece \\ (Reçu le 2 avril 1984, révisé le 17 juillet, accepté le 25 septembre 1984)
}

\begin{abstract}
Résumé. - On présente une méthode simplifiée pour le calcul du potentiel moyen d'interaction d'un ion avec une molécule polaire aux énergies thermiques de collision. En appliquant ce potentiel et les critères de Langevin aux collisions de capture on dérive une expression pour la constante de vitesse de la réaction. Cette constante théorique de la vitesse est comparée à plusieurs données expérimentales, aux résultats de calculs récents de trajectoires et aux prévisions d'autres théories. Il est démontré qu'elle est en bon accord avec l'expérience et qu'elle est proche d'autres modèles basés sur des méthodes statistiques ou variationnelles. Elle est aussi en bon accord avec des calculs détaillés de trajectoires.

Abstract. - A simplified method is presented to compute the average interaction potential of an ion reacting with a dipolar molecule at thermal collision energies. Using this potential and applying the Langevin criteria for capture collisions, an expression for the reaction rate constant is derived. The theoretical rate constant is compared with many experimental data, the results of recent trajectory calculations and the predictions of other theories. It is shown to be in good agreement with experiment and it is found close to other models based on statistical or variational methods. It is also found in good agreement with detailed trajectory calculations.
\end{abstract}

\section{Introduction.}

Since the time of the Langevin formulation of the polarization theory [1], which gave rise to the well known ion-molecule capture collision rate constant,

$$
k_{\mathrm{L}}=2 \pi q(\alpha / m)^{1 / 2}
$$

much work has been carried out in this subject. Particular interest has been focussed to an important class of this type of processes which includes the reactions between an ion and a polar molecule. When the neutral molecule possesses a permanent dipole moment, $\mu$, the additional interaction between the ion and the dipole, $V(r, \vartheta)=-\left(q \mu / r^{2}\right) \cos \vartheta$, plays a very important role in the reaction dynamics. Detailed trajectory calculations have shown [2] that the magnitude of the rate constant is seriously affected by the presence of the permanent dipole moment. Thus much effort was devoted to investigate the effect of the ion-dipole potential and several models were proposed which account for the description of the reaction and the magnitude of the rate constant with variable success [3-5].

A first significant attempt to account for the effect of the ion-dipole potential was the ADO (Average Dipole Orientation) theory, suggested by Bowers and coworkers [6]. In their model 
the probability $P(\vartheta ; r)$ that a dipole is found at an orientation $\vartheta$ with respect to the incident ion at a distance $r$ is assumed to be

$$
P(\vartheta ; r) \propto \frac{\sin \vartheta}{\dot{\vartheta}} \propto \frac{\sin \vartheta}{\left[E_{\mathrm{rot}}+\left(q \mu / r^{2}\right) \cos \vartheta\right]^{1 / 2}},
$$

where $\dot{\vartheta}$ is the angular velocity of the dipole in the plane of the collision, $E_{\text {rot }}$ is the thermal rotational energy of the polar molecule and $\sin \vartheta$ is a spatial orientation factor accounting for the number of ways the dipole can be oriented with an angle $\vartheta$ with respect to the line of centres of the collision. The average value of the $\cos \vartheta$ at a particular stparation $r$ is then

$$
\langle\cos \vartheta(r)\rangle=\int \cos \vartheta P(\vartheta ; r) \mathrm{d} \vartheta / \int P(\vartheta ; r) \mathrm{d} \vartheta \text {. }
$$

The ADO theory was parametrized in a simple form to allow easy comparison with experiment and it was found to predict most experimental values with a good degree of accuracy. Despite its success the ADO theory is still not satisfactory for many ion-dipole systems. The reason lies on the fact that it underestimates usually the magnitude of the experimental rate constant. This is contradictory for a theory which calculates a collision rate constant and which therefore should lead to an upper bound to the experimental reaction rate, due to possible steric effects. The contradiction was recognized by the same workers who tried to account for it in various ways [7]. Lately a variational transition state theory was developed with good success [9]. This theory is formulated in terms of a microcanonical ensemble and the assumed ion-molecule separation in the transition state is varied until a minimum rate constant is found which is taken as the reaction rate constant. The minimum value corresponds to the best potential energy surface between reactants and products.

Barker and coworkers followed a different approach [8]. They " preaveraged » the ion-dipole interaction energy $V(r, \vartheta)$ over the Boltzmann distribution of energy to obtain

$$
V(r)=\frac{\int_{0}^{\pi} \exp \left(-V(r, \vartheta) / k T_{\mathrm{R}}\right) V(r, \vartheta) \sin \vartheta \mathrm{d} \vartheta}{\int_{0}^{\pi} \exp \left(-V(r, \vartheta) / k T_{\mathrm{R}}\right) \sin \vartheta \mathrm{d} \vartheta}=\frac{\mu q}{r^{2}} \mathcal{L}\left(\frac{q \mu}{r^{2} k T_{\mathrm{R}}}\right)
$$

where $T_{\mathrm{R}}$ is the rotational temperature of the molecule and $\mathcal{L}(x)$ is the Langevin function. This theory agrees well with the absolute rate constants for momentum transfer processes but it largely overestimates the experimental rate constants for reactions. Recently a thermodynamic approach was developed $[10,11]$ in correlation with the statistical formulation of equation (4). As it is known from electrostatics the amount of work necessary to increase a charge distribution by certain amount is expended in a reversible process and consequently it is equal to the change in work content or Helmholtz free energy, $F$, under constant volume and temperature [12]. Capture rate constants were obtained from the free energy function of the form

$$
F(r, t)=-k T \ln Q
$$

where $Q$ may be considered as the partition function of a gas of independent dipoles, in which an orientation factor per dipole is included :

$$
Q=\left(k T r^{2} / \mu q\right) \sinh \left(\mu q / k T r^{2}\right) .
$$

This approach is also in good agreement with experiment and in good agreement with the variational transition state theory. Recently other theories have also appeared with good success $[15,16]$. 


\section{Present theory.}

The ADO theory was the first approach to account successfully for the effect of the permanent dipole moment on the macroscopic rate constant. It is formulated in terms of an $r$-dependent average of the ion-molecule interaction over the orientation angle $\vartheta$. Although the resulting potential includes the concept of an averaging procedure it still represents in a phenomenological way the most probable conditions under which single collisions occur, because in most cases the value of $\cos \vartheta$ will indeed be close to the average value. The ADO theory then accounts for single collision processes and imposes the capture criteria in much the same way as is done in polarization theory. Other theories developed later consider the thermal ion-dipole system in a more statistical sense or they base their formulation on the concept of the ensemble. Thus they loose the advantage to base their method on a simple, pure collision treatment and calculate the macroscopic rate constant from the properties of the reaction at the microscopic level. We think that the ADO theory starts from the right principle to try to compute an average value of $\cos \vartheta$ as a function of the intermolecular distance $r$. We disagree though in the way this average is calculated. The ADO model averages $\cos \vartheta$ over all values of the rotational energy, $E_{\text {rot }}$, and then computes a capture cross section. We suggest that better results may be obtained by computing the cross section for each value of $E_{\text {rot }}$ and then averaging the result over a thermal distribution. The resulting thermal rate constants are typically $10 \%$ larger than the ADO results and in better agreement with experiment [13].

In the present approach we assume that all polar molecules whose rotational energies are higher than the ion-dipole interaction energy will continue complete rotation. The other molecules will rotate up to a maximum value of the orientation angle $\vartheta_{\max }$. Assuming also that no transfer of angular momentum occurs, the maximum value of $\vartheta$ may be determined as a function of $E_{\text {rot }}$ and $r$ from the equation

$$
\begin{aligned}
\cos \vartheta_{\max } & =-\left(r / r_{0}\right)^{2} \\
r_{0} & =\left(q \mu / E_{\mathrm{rot}}\right)^{1 / 2} .
\end{aligned}
$$

Next, we calculate the average value of $\cos \vartheta$ as a function of $E_{\text {tot }}$ and $r$. To do so we distinguish two cases :

$2.1 r_{0}<r<\infty$. - This region corresponds to a rotational energy larger than the ion-dipole interaction, i.e., to free rotation according our assumption. We consequently accept the average value of $\cos \vartheta$ to be zero.

$$
\langle\cos \vartheta\rangle=0 \text { for } r_{0}<r<\infty \text {. }
$$

The interaction energy reduces to the polarization potential $V(r)=-\left(q \alpha^{2} / 2 r^{4}\right)$ where $\alpha$ is the polarizability of the neutral molecule. The capture cross section is calculated following the Langevin formulation. By including the centrifugal potential which results from the orbital angular momentum of the system, we obtain an effective potential

$$
V_{\mathrm{eff}}(r)=-q \alpha^{2} / 2 r^{4}+E b^{2} / r^{2},
$$

where $b$ is the impact parameter and $E=\frac{1}{2} m v^{2}$ is the initial relative kinetic energy of the system. When the initial relative kinetic energy is lower than the barrier height, the trajectory will be pushed away from the potential barrier and the collision will be a distant one. When $E$ is higher than the barrier height the collision partners approach each other with acceleration and come into close contact. There is a critical impact parameter $b_{c}$ for each value of $E$ for which $E$ at least equals the potential maximum. This corresponds to the classical turning point $r_{\mathrm{c}}$ where the 
instantaneous relative velocity vanishes. Therefore $b_{\mathrm{c}}$ may be determined from the conditions

$$
\begin{gathered}
E \stackrel{\dot{=}}{=} V_{\text {eff }}(r) \\
{\left[\partial V_{\mathrm{eff}}(r) / \partial r\right]_{r=r_{\mathrm{c}}}=0}
\end{gathered}
$$

and it is found to be

$$
b_{\mathrm{c}}=\left(2 q^{2} \alpha / E\right)^{1 / 2} .
$$

Finally the capture cross section is calculated from

$$
\sigma_{1}(v)=\pi b_{\mathrm{c}}^{2}=\pi q(2 \alpha / E)^{1 / 2} .
$$

Equation (12) gives us the value of the capture cross section $\sigma_{1}(v)$ for the ion-dipole collision for which $r_{0}<r<\infty$. Because the separation $r_{\mathrm{c}}$ must be larger than $r_{0}$, equation (12) is valid for the ion-dipole systems for the following range of values of the initial relative kinetic energy :

$$
E<\alpha E_{\mathrm{rot}}^{2} / 2 \mu^{2} .
$$

$2.2 \underline{0<r<r_{0}}$. - In this case we assume that $\langle\cos \vartheta\rangle$ is given by

$$
\langle\cos \vartheta\rangle=\frac{\int_{0}^{\vartheta_{\max }} \cos \vartheta \sin \vartheta \mathrm{d} \vartheta}{\int_{0}^{9_{\max }} \sin \vartheta \mathrm{d} \vartheta}=\frac{1}{2}\left[1-\left(\frac{r}{r_{0}}\right)^{2}\right] \text {, }
$$

where $\sin \vartheta$ is a spatial orientation factor. The average effective potential now becomes

$$
V_{\mathrm{eff}}\left(r, E_{\mathrm{rot}}\right)=E b^{2} / r^{2}-q^{2} \alpha / 2 r^{4}-q \mu / 2 r^{2}+\frac{1}{2} E_{\mathrm{rot}} .
$$

Using this potential and applying again the Langevin criteria, equation (10), we find that

for

$$
\sigma_{2}(v)=\frac{\pi q \mu}{E_{\mathrm{rot}}}+\frac{\pi \alpha q E_{\mathrm{rot}}}{2 \mu E}
$$

and

$$
\frac{\alpha E_{\mathrm{rot}}^{2}}{2 \mu^{2}}<E<\frac{\alpha E_{\mathrm{rot}}^{2}}{2 \mu^{2}}+\frac{1}{2} E_{\mathrm{rot}}
$$

$$
\sigma_{3}(v)=\frac{\pi q \mu}{2 E}+\pi\left(\frac{2 \alpha q^{2}}{E}\right)^{1 / 2}\left(E-\frac{E_{\mathrm{rot}}}{2 E}\right)^{1 / 2}
$$

for

$$
E>\frac{\alpha E_{\mathrm{rot}}^{2}}{2 \mu^{2}}+\frac{1}{2} E_{\mathrm{rot}} .
$$

For all values of the initial relative kinetic energy the resulting cross section is

$$
\sigma_{\mathrm{c}}\left(v, E_{\mathrm{rot}}\right)=\sigma_{1}\left(v, E_{\mathrm{rot}}\right)+\sigma_{2}\left(v, E_{\mathrm{rot}}\right)+\sigma_{3}\left(v, E_{\mathrm{rot}}\right) .
$$

We see that the capture cross section $\sigma_{\mathrm{c}}$ is a function of both the initial relative kinetic energy $E$ 
and the initial rotational energy $E_{\text {rot }}$ of the polar molecule. The macroscopic thermal rate constant may be calculated by

$$
k\left(T, E_{\mathrm{rot}}\right)=\int_{0}^{\infty} v \sigma_{\mathrm{c}}\left(v, E_{\mathrm{rot}}\right) P(v ; T) \mathrm{d} v
$$

where $P(v ; T)$ is the Boltzmann thermal velocity distribution. Substituting into equation (21) and performing the average over the thermal velocity distribution we obtain the rate constant as a function of the temperature and the initial rotational energy. We then average $k\left(t, E_{\text {rot }}\right)$ over the rotational energy distribution to obtain

$$
\begin{aligned}
k(z)=2 & \pi q\left(\frac{\alpha}{m}\right)^{1 / 2}\left\{\frac{2}{3}-\frac{2}{3} \exp \left(1.125 z^{2}\right)[1-\operatorname{erf}(1.061 z)]+\right. \\
& \left.+\exp \left(z^{2} / 2\right)[1-\operatorname{erf}(0.707 z)]+(2 / \pi)^{1 / 2} z \int_{0}^{\infty}\left(\frac{1-\mathrm{e}^{x / 2}}{x}\right) \exp \left(-x^{2} / 2 z\right) \mathrm{e}^{-x} \mathrm{~d} x\right\},
\end{aligned}
$$

where $\operatorname{erf}(x)$ is the error function and $2 \pi q(\alpha / m)^{1 / 2}$ is the Langevin rate constant $k_{\mathrm{L}}$ of equation (1). The rate constant is expressed in terms of the dimensionless parameter $z=(\mu / \alpha k T)^{1 / 2}$, which is the suitable variable for the description of these systems. Equation (22) may be solved numerically for any value of $z$ to derive the rate constant of the capture ion-dipole molecule collisions.

We may summarize the calculation described here in the following steps : (1) determine the angle-averaged intermolecular potential between an ion and a rotating dipole as a function of the initial rotational energy of the dipole, $E_{\text {rot }}$, the initial relative translational energy, $E$, and the distance between the ion and the centre of mass of the dipole, $r$; $(2)$ calculate the capture cross section as a function of $E_{\text {rot }}$ and $E$; (3) integrate the product of the cross section and the relative velocity over the Boltzmann distribution of relative velocities to obtain the rate constant as a function of $T$ and $E_{\text {rot }} ;(4)$ integrate over the rotational energy distribution to obtain the reaction rate constant as a function of the temperature.

\section{Results and discussion.}

Before any comparison with experiment is made the main assumptions of the theory will be summarized. Thermal rate constants are calculated for ion-polar molecule exothermic reactions with no activation energy barrier. While it is true that for most ion-molecule reactions the activation energy barrier is small, this is not always the case. Some reactions have large energy barriers and the experimental rate constants are considerably lower than the Langevin value. Also all models assume that reaction occurs with unit probability at every close collision. However, steric effects may reduce the reaction probability. Thus the collisional capture rate constants must be an upper bound to the reaction rate constant.

In figure 1 the predictions of the various theories are tested against experimental rate constants at a temperature of $297 \mathrm{~K}$ for exothermic proton transfer reactions of the type

$$
\mathrm{XH}^{+}+\mathrm{Y} \rightarrow \mathrm{YH}^{+}+\mathrm{X}
$$

where $\mathrm{Y}$ is the dipolar molecule. The various theories include the ADO model, the statistical thermodynamic approach, the variational transition state theory and the adiabatic invariance approach [15], as well as the present approach. Comparison with Sakimoto's results is not straightforward and thus not included. The experimental data are for the following dipolar molecules : $\mathrm{HCN}, z=9.10$ [17], $\mathrm{NH}_{3}, z=4.80$ [18, 19], $\mathrm{H}_{2} \mathrm{O}, z=7.53$ [20] and $\mathrm{CH}_{2} \mathrm{O}, z=6.86$ [21]. We note that the ADO theory yields unexpectedly poor results. The other three theories are in good agreement with experiment and with each other. The present treatment starting from similar 
principles as the ADO method is found in better agreement with experiment and closer to the other three theories mentioned above. Bates gave an explanation [14] to the failure of the ADO method, based on the wrong limiting behaviour of $\cos \vartheta$ for very small values of $E_{\text {rot }}$. As the rotational energy goes to zero, elementary physical considerations show that $\cos \vartheta$ must be unit, the dipole being completely locked. The ADO leads to the relation

$$
\langle\cos \vartheta\rangle=\frac{1}{3}\left(1-E_{\mathrm{rot}}(\infty) r^{2} / q \mu\right)
$$

where $E_{\text {rot }}(\infty)$ is the rotational energy of the dipolar molecule at $r=\infty$. Clearly when $E_{\text {rot }}(\infty)$ tends to zero, $\langle\cos \vartheta\rangle$ does not take the proper limiting value. In our theory though $\langle\cos \vartheta\rangle$ as expressed in equation (7) shows the correct limiting behaviour.

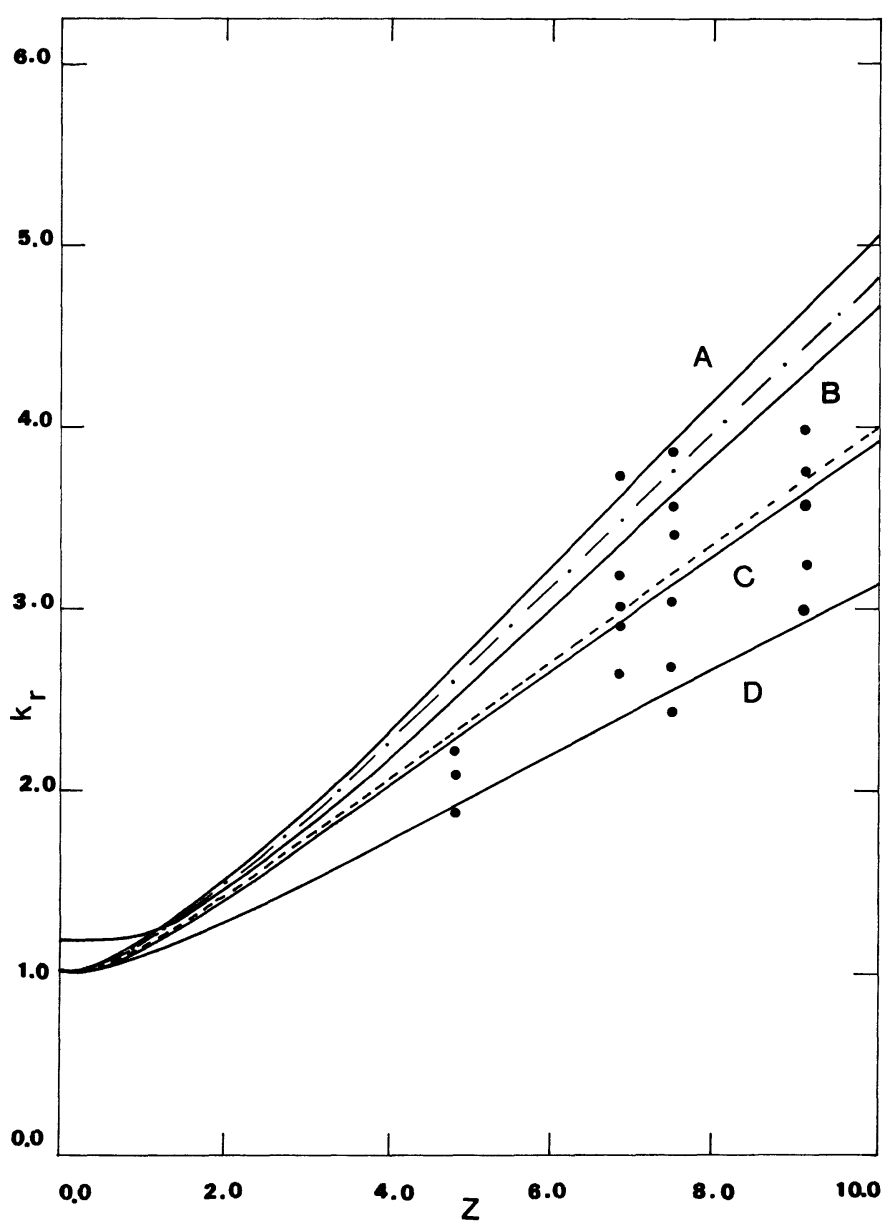

Fig. 1. - Plot of the reduced rate constant $k_{\mathrm{r}}=k(z) / k_{\mathrm{L}}$ vs. the reduced dipole moment $z$. Line A corresponds to statistical method, line B to variational method and D to the ADO method. The dot-dashed line between A and $B$ corresponds to the adiabatic invariance approximation, line $C$ represents the present theory and the dashed line is the result of trajectory calculations. All lines but the present theory are read from graphs. The dots represent experimental data. See text for details. 
In figure 1 we have also included the results of detailed trajectory calculations [22]. They are practically identical to our results and they greatly support our method.

In summary we may say that the present approximation to the ion-dipole problem yields an analytical expression for the rate constant which is in good agreement with experiment and in good agreement with the results of detailed trajectory calculations.

\section{References}

[1] Gioumousis, G. and Stevenson, D. P., J. Chem. Phys. 29 (1958) 294.

[2] Dugan, J. V., JR. and Magee, J. L., J. Chem. Phys. 47 (1967) 3103.

[3] Moran, T. F. and Hamill, W. H., J. Chem. Phys. 39 (1963) 1413.

[4] Dugan, J. V., Jr., Chem. Phys. Lett. 21 (1973) 476.

[5] Bowers, M. T. and Laudenslager, J. B., J. Chem. Phys. 56 (1972) 4711.

[6] Su, T. and Bowers, M. T., J. Chem. Phys. 58 (1973) 3027 ;

Bass, L., Su, T., Chesnavich, W. J. and Bowers, M. T., Chem. Phys. Lett. 34 (1975) 119.

[7] Su, T., Su, E. C. F. and Bowers, M. T., J. Chem. Phys. 69 (1978) 2243.

[8] Barker, R. A. and Ridge, D. P., J. Chem. Phys. 64 (1976) 4411.

[9] Chesnavich, W. J., Su, T. and Bowers, M. T., J. Chem. Phys. 72 (1980) 2641.

[10] TUrulski, J. and Forys, M., J. Phys. Chem. 83 (1979) 2815.

[11] Celli, F., Weddle, G. and Ridge, D. P., J. Chem. Phys. 73 (1980) 801.

[12] BotTCher, C. J. F., Theory of Electric Polarization (Elsevier, Amsterdam) 1973.

[13] Kosmas, A. M., Ph. D. Thesis, University of Illinois at Chicago (1980).

[14] Bates, D. R., Chem. Phys. Lett. 82 (1981) 396.

[15] Bates, D. R., Chem. Phys. Lett. 97 (1983) 19.

[16] Sakimoto, K., Chem. Phys. 79 (1983) 137.

[17] Mackay, G. I., Betowski, L. D., Paysant, J. D., Schiff, H. I. and Bohme, D. K., J. Phys. Chem. 80 (1976) 2919.

[18] Hemsworth, R. M., Paysant, J. D., Schiff, H. I. and Bohme, D. K., Chem. Phys. Lett. 26 (1974) 417.

[19] Lindinger, W., Albritton, D. L., Fehsenfeld, F. C., Schmeltekopf, A. L. and Ferguson, E. E., J. Chem. Phys. 62 (1975) 3549.

[20] Betowski, L. D., Paysant, J. D., Mackay, G. I. and Bohme, D. K., Chem. Phys. Lett. 31 (1975) 321.

[21] Tanner, S. D., MaCKay, G. I. and Bohme, D. K., Can. J. Chem. 57 (1979) 2350.

[22] Su, T. and Chesnavich, W. J., J. Chem. Phys. 76 (1982) 5183. 